\title{
Mesoscopic proximity effect probed through superconducting tunneling contacts
}

\author{
Frank K. Wilhelm ${ }^{1,2}$ and Alexander A. Golubov ${ }^{3}$ \\ ${ }^{1}$ Institut für Theoretische Festkörperphysik, Universität Karlsruhe, D-76128 Karlsruhe, Germany \\ ${ }^{2}$ Quantum Transport Group, TU Delft, P.O. Box 5046, 2600 GA Delft, The Netherlands \\ ${ }^{3}$ Department of Applied Physics, University of Twente, 7500 AE Enschede, The Netherlands
}

(Received 31 January 2000)

\begin{abstract}
We investigate the properties of complex mesoscopic superconducting-normal hybrid devices, Andreev Interferometers in the case where the current is probed through a superconducting tunneling contact whereas the proximity effect is generated by a transparent $S N$ interface. We show within the quasiclassical Green'sfunctions technique, how the fundamental SNIS element of such structures can be mapped onto an effective $S^{\prime} I S$ junction, where $S^{\prime}$ is the proximized material with an effective energy gap $E_{g}<\Delta$. The conductance through such a sample at $T=0$ vanishes if $V<\Delta+E_{g}$, whereas at $T>0$ the conductance shows a peak at $V$ $=\Delta-E_{g}$. We propose the Andreev interferometer, where $E_{g}$ can be tuned by an external phase $\phi$ and displays maxima at $0 \bmod 2 \pi$ and minima at $\pi \bmod 2 \pi$. This leads to peculiar current-phase relations, which depart from a zero-phase maximum or minimum depending on the bias voltage and can even show intermediate extreme at $V \approx \Delta$. We propose an experiment to verify our predictions and show how our results are consistent with recent, unexplained experimental results.
\end{abstract}

The proximity effect, although already known for many decades (see, e.g., Ref. 1), has recently attracted renewed scientific interest in the context of mesoscopic normalsuperconducting hybrid structures, which are now experimentally acessible due to progress in nanofabrication and measurement support technology. ${ }^{2-4}$ Departing from the properties of single junction and the nonmonotonic diffusion conductance of $S N$ wires, the interest turned to the possibility of tuning the conductance by an external phase or a loop in the normal part. On the other hand, if probed through tunneling contacts ${ }^{5}$ the conductance is controlled by the DOS and the induced minigap, ${ }^{6,7}$ which can also be controlled by a phase ${ }^{8}$ and hence opens another channel for phase controlled conductance of a different sign. ${ }^{9,10}$ If a system contains more than one superconducting terminal, a supercurrent can flow. The situation becomes more difficult and, in particular, time dependent, if nonequilibrium is created by applying an external voltage parallel to the junction.

This latter situation is substantially simplified, if one of the contacts is separated from the rest of the structure by a tunneling barrier. In that case, the voltage and phase drop is concentrated at the barrier and the problem is essentially split into two parts: The time dependence of the phase at the contact and the proximity effect, which determines the superconducting properties at the normal side of the contact, within the normal metal. In that case, the physics should be basically identical to the case of an $S^{\prime} I S$ junction, where the properties of the "superconductor" $S^{\prime}$ are entirely controlled by the proximity effect, i.e., we expect a gap of size $E_{g}<\Delta$ where, if the junction is long, $d \gg \xi_{0} E_{g} \propto E_{\mathrm{Th}}=D / d^{2}$, the Thouless energy. Hence we will expect the known ${ }^{11}$ physics of such $S^{\prime} I S$ contacts: The onset of a tunneling current at $V=\Delta+E_{g}$ at any $T$ plus the appearance of a current peak at $V=\Delta-E_{g}$ if $T>0$. The origin of this peak can be easiest understood within a semiconductor representation of the two superconductors, see, e.g., Ref. 12.
Such a mesoscopic structure can be manufactured in a controlled manner, which, to the best of our knowledge, has not yet been done. Nevertheless, we are going to discuss the connection to two experiments: Kutchinsky et al. ${ }^{13}$ studied the conductance in a T-shaped interferometer with superconducting contacts in a semiconducting systems, where unwanted barriers at the interfaces are likely to occur. Antonov et $a .^{9}{ }^{9}$ in turn, studied a sample with normal tunneling contacts, which might eventually be connected to superconducting pieces.

Model and basic equations. Mesoscopic proximity systems are efficiently and quantitatively described by the quasiclassical Green's-functions technique, described in Ref. 14 and references therein $(4-6,49$, and 50) as well as in Ref. 15. In this approach, the microscopic Gor'kov equation is reduced to the more handy Usadel equation by various systematic approximations. At interfaces, this equation is supplemented by boundary conditions (in our case, deviations from these conditions as discussed in Ref. 4 are not likely to occur)

$$
\begin{gathered}
p_{F 1}^{2} l_{1} \hat{G}_{1} \frac{d}{d x} \hat{G}_{1}=p_{F 2}^{2} l_{2} \hat{G}_{2} \frac{d}{d x} \hat{G}_{2}, \\
l_{2} \hat{G}_{2} \frac{d}{d x} G_{2}=t\left[\hat{G}_{2}, \hat{G}_{1}\right] .
\end{gathered}
$$

These conditions guarantee current conservation. We apply them to the case of small transparencies $t \ll 1$, which enforce that the drop of phase and voltage is concentrated at the insulating layer. ${ }^{6,16}$ The current can thus be expressed by a tunneling formula

$$
\begin{gathered}
J=\operatorname{Re} J_{p}(V, T) \sin \phi+\operatorname{Im} J_{p}(V, T) \cos \phi+\operatorname{Im} J_{q}(V, T), \\
\phi=2 e V t+\phi_{0}
\end{gathered}
$$




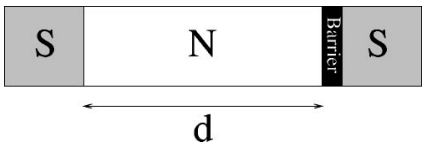

FIG. 1. Asymmetric SNIS junction.

for the current through the interface. Here, the quasiparticle tunneling current amplitude is

$$
\begin{aligned}
\operatorname{Im}\left[J_{q}(V, T)\right]= & \frac{G_{n}}{2 e} \int d E \operatorname{Re}\left[G_{N}^{R}(E)\right] \operatorname{Re}\left[G_{B C S}^{R}(E+V)\right] \\
& \times\left[\tanh \left(\frac{E+e V}{2 T}\right)+\tanh \left(\frac{E}{2 T}\right)\right]
\end{aligned}
$$

and $\operatorname{Re} G^{R}$ gives the quasiparticle DOS. At a given dc voltage bias $V \neq 0$, it is the only contribution to the dc current component. $V$ is the externally applied voltage. This is consistent with the assumption of a barrier resistance much higher than the diffusion resistance of the normal metal $R_{T}$ $\gg R_{N}$. It also implies, that the proximity-enhanced diffusion conductivity, which is extensively discussed in recent literature, ${ }^{14,15}$ gives an effect of the order of $R_{N}$ and can be neglected in this limit. The interplay of both phenomena in experimental setups will be discussed later on. We want to apply this result to the specific case of an SNIS junction, see Fig. 1. Equation (3) allows to identify this system with an effective $S^{\prime} I S$-Josephson junction, where the "superconductor" $S^{\prime}$ is the normal-metal layer influenced by the proximity effect. We can characterize $S^{\prime}$ by the Green's functions at the interface calculated from the Usadel equation assuming — in order to be consistent with $R_{\mathrm{T}} \gg R_{\mathrm{N}}$ - a highly resistive interface and consequently a vanishing phase drop over the $N$ part. The "superconductor" $S^{\prime}$ has a gap of size $E_{\mathrm{G}} \sim \min \left(E_{\mathrm{Th}}, \Delta\right)$, see Fig. 2. Thus we expect from a semiconductor model that the system shows a dc supercurrent at $V=0$ and a dc quasiparticle current at $V \geqslant \Delta+E_{\mathrm{G}}$. Moreover, at finite temperature, a few empty states below $E_{\mathrm{F}}$ and a few quasiparticles above $E_{\mathrm{F}}$ are available, enabling transport already at $V \geqslant \Delta-E_{\mathrm{G}}$ [see Eq. (4)] hence leading to a

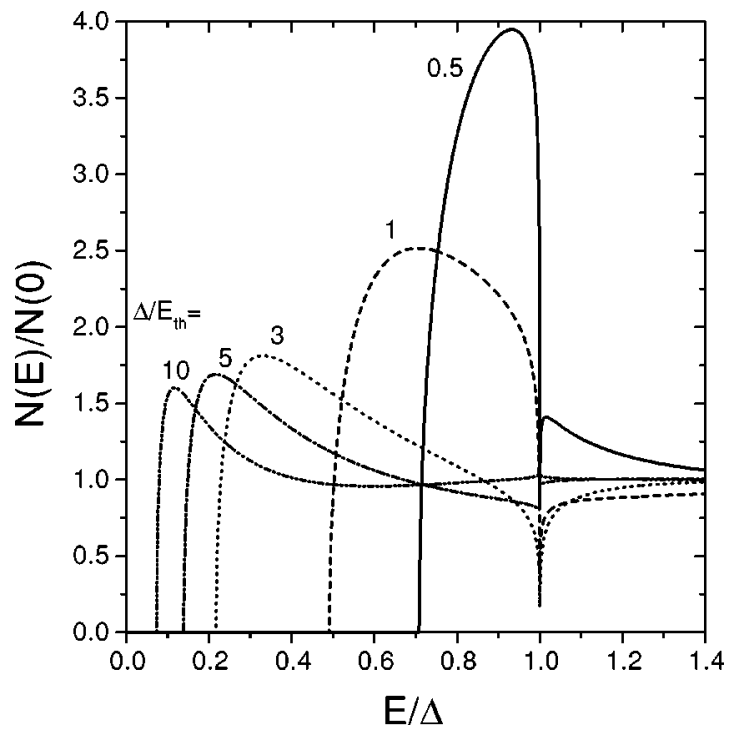

FIG. 2. DOS in the normal metal at the interface.

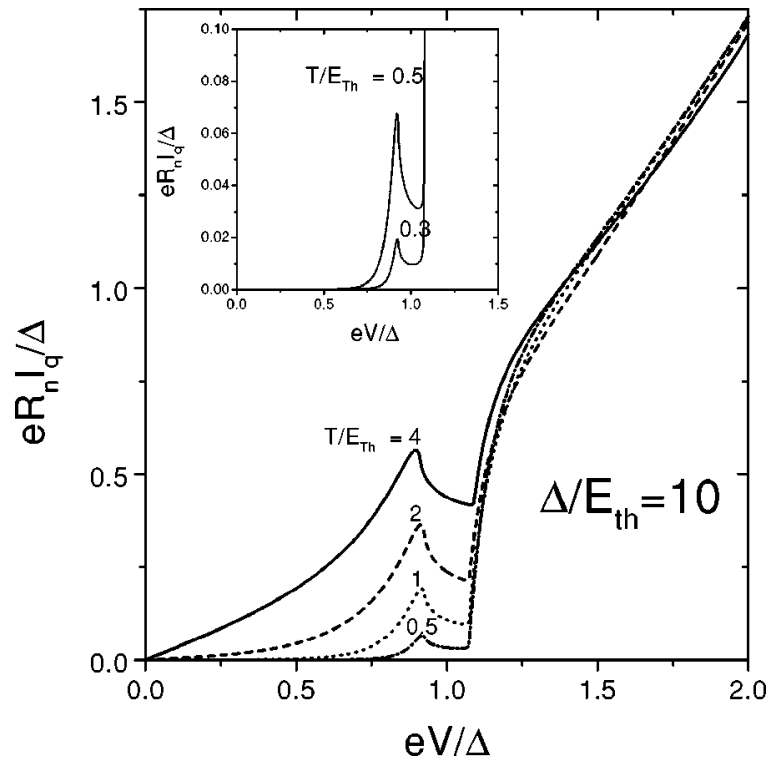

FIG. 3. $I-V$ characteristics of an $S N I S$ junction for $E_{\mathrm{Th}}=0.1 \Delta$; the inset shows the same figure on a larger scale.

logarithmic quasiparticle current peak there. ${ }^{17}$ Unlike the situation in a massive superconductor, the induced DOS in $S^{\prime}$ does not diverge at the gap edge but has a maximum slightly above $E_{G}$, see Fig. 2, thus we can conclude that also the peak will be smoothened and be slightly above $\Delta-E_{\mathrm{G}}$. Additionally, due to BCS singularity in $S$, another structure is present in DOS of $S^{\prime}$ at $E \sim \Delta$, which is weakened with increasing thickness $d$ (or decreasing Thouless energy).

Numerical results. In order to obtain quantitative results from Eqs. (3) and (4), the function $\operatorname{Im}\left[G^{R}(d)\right]$ has to be calculated. It is given by the solution of the Usadel equation $D \partial_{x}^{2} \alpha^{R}=2 i E \sinh \alpha^{R}$ with boundary conditions $\alpha^{R}(x=0)$ $=\alpha_{S}^{R}=\operatorname{Atanh}|\Delta / E|$ at the superconductor and $\left.\partial_{x} \alpha^{R}\right|_{x=d}=0$ at the tunneling barrier, through $G^{R}(d)=\cosh \alpha^{R}$. These nonlinear equations are in general not solvable analytically. Nevertheless, we find from a low-energy expansion following the

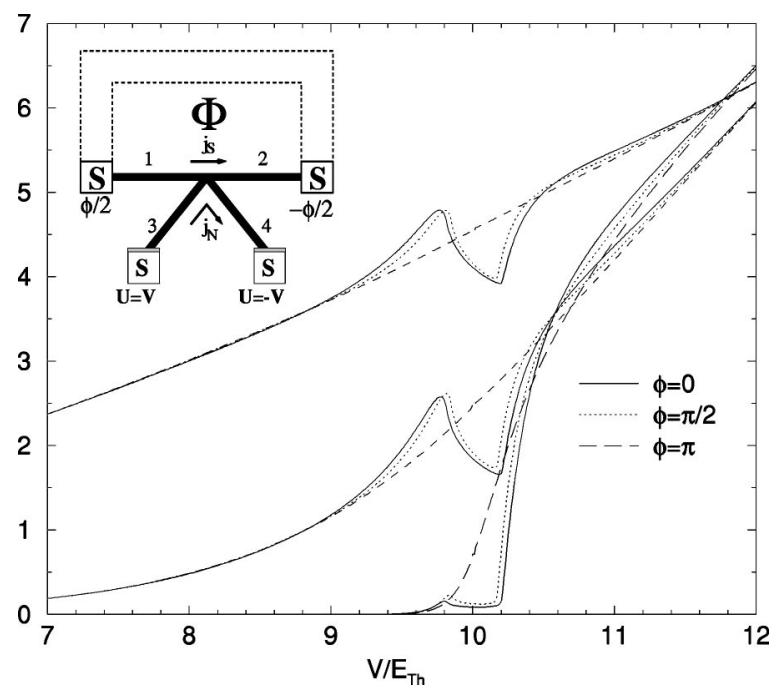

FIG. 4. $I-V$ characteristics of the interferometer shown in the inset for $\Delta=10 E_{\mathrm{Th}}$ at different values of $\phi$. Temperatures are (top to bottom) $T=5,1,0.1 E_{\mathrm{Th}}$. All interferometer arms are assumed to be of the same length. 
lines of Ref. 18 that $\operatorname{Im}\left[\alpha^{R}\right]=0$ to all orders, which indicates the presence of a gap in the spectrum with a sharp edge (at the convergence radius of the low-energy expansion). At high energies, $E \gg E_{\mathrm{Th}}$, the system is decoupled from the boundary conditions at the barrier and $\alpha(d)$ $=4 \mathrm{~A} \tanh \left(\tanh \left(\alpha_{S} / 4\right) \exp -\sqrt{-2 i E / E_{T h}}\right)$ indicating that the deviation from the normal-state value is exponentially cut off at those energies. This is consistent with our numerical result, see Fig. 2.

Our qualitative predictions in the preceding section are confirmed by our numerical results, Fig. 3. As predicted, the peaks grow and smear out with increasing temperature, but stay visible up to temperatures far above $E_{\mathrm{Th}}$. Furthermore, the feature becomes more pronounced if $E_{G}$ is big, i.e., for a shorter junction.

SNIS Andreev interferometers. Even if this type of junction is not prepared on purpose, during the fabrication process an asymmetric barrier can easily show up accidentally, e.g., if the $N$ metal is a highly doped semiconductor and a Schottky barrier is likely to occur or if the structure is prepared out of two layers within a two-step shadow evaporation technique. ${ }^{19}$ In this situation, the limit $r \gg 1$ may not be perferctly achieved and corrections to the behavior we discuss may occur. These correcctions may be twofold: (i) a softening of the induced gap giving an additional rounding of the structure and (ii) the appeearence of the phase-coherent
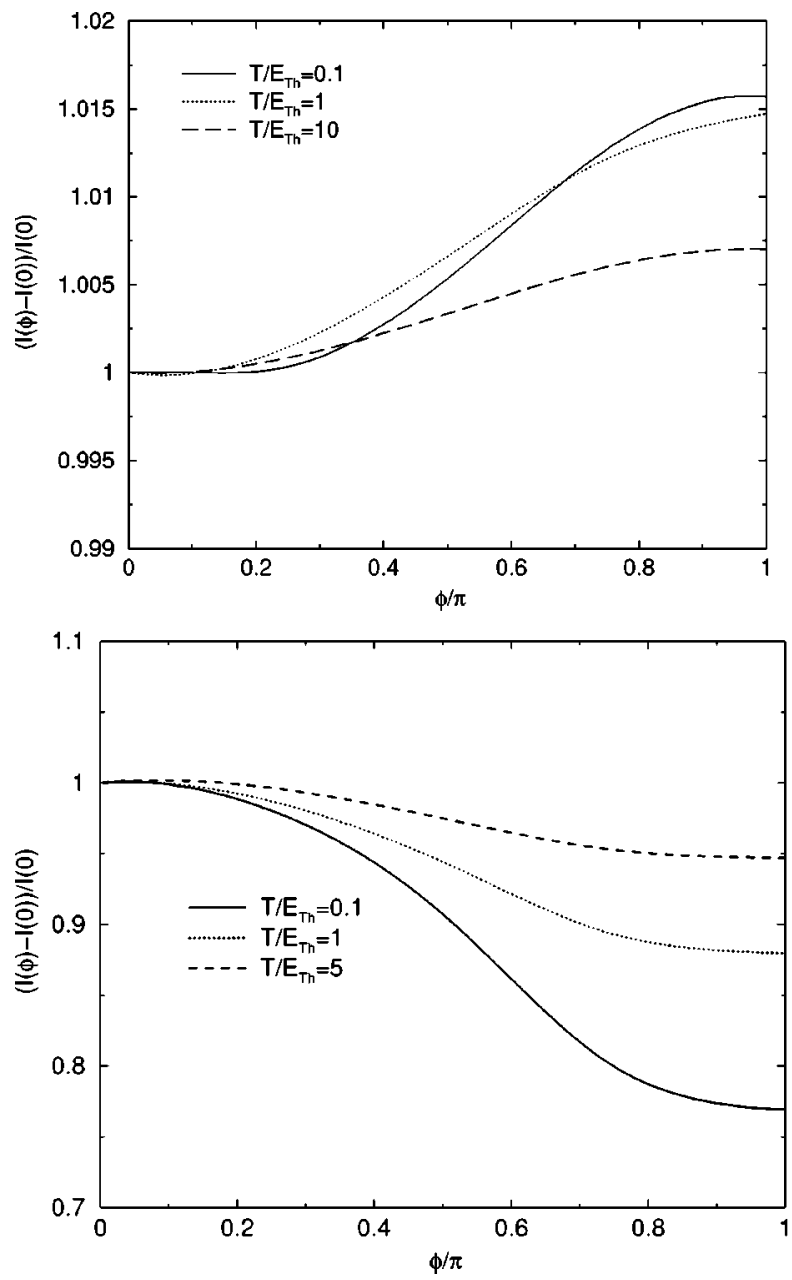

correction to the diffusive conductivity. The latter changes the conductivity at low voltages $V \simeq E_{\mathrm{Th}}$ by at most a factor of 2 and can hence be easily discriminated from the very pronounced phenomenon we describe which happens at $V$ $\simeq \Delta$, i.e., a usually much higher voltage.

As a model, we consider the interferometer Fig. 4 discussed already in Ref. 9 in the case when the tunneling barriers are strong and all four reservoirs are superconducting. The phase difference allows to control the strength of the proximity effect, manifested here in the size of the minigap $E_{\mathrm{G}}(\phi)$, which varies between $E_{G}^{\max }$ at even and 0 at odd multiples of $\pi$. The influence of the phase difference in the interferometer is hence most pronounced for $\left|\Delta-E_{\mathrm{G}}^{\max }\right| \leqslant V$ $\leqslant\left|\Delta+E_{\mathrm{G}}^{\max }\right|$. The $I-V$ characteristics at a fixed phase, Fig. 4 resembles the form already discussed in Fig. 3 but is slightly smoothened. At fixed temperatures and voltages, the $I-\phi$ relation shows many shapes including zero-field minima and maxima as well as additional extrema at intermediate phases, see Fig. 5. This can be traced back to the motion of $E_{\mathrm{G}}(\phi)$ : At $V<\Delta-E_{G}^{\max }$, a bigger gap slightly lowers the current (see left upper in Fig. 5), at $\Delta-E_{G}^{\max }<V<\Delta$, we are in the vicinity of the induced peak, which only shows up due to $E_{g}$, so the current is rather suppressed by shifting the gap (see right upper in Fig. 5). At $\Delta<V<\Delta+E_{G}^{\max }$, the situation is more subtle: The current will be maximum, if the edge at $\Delta+E_{G}$
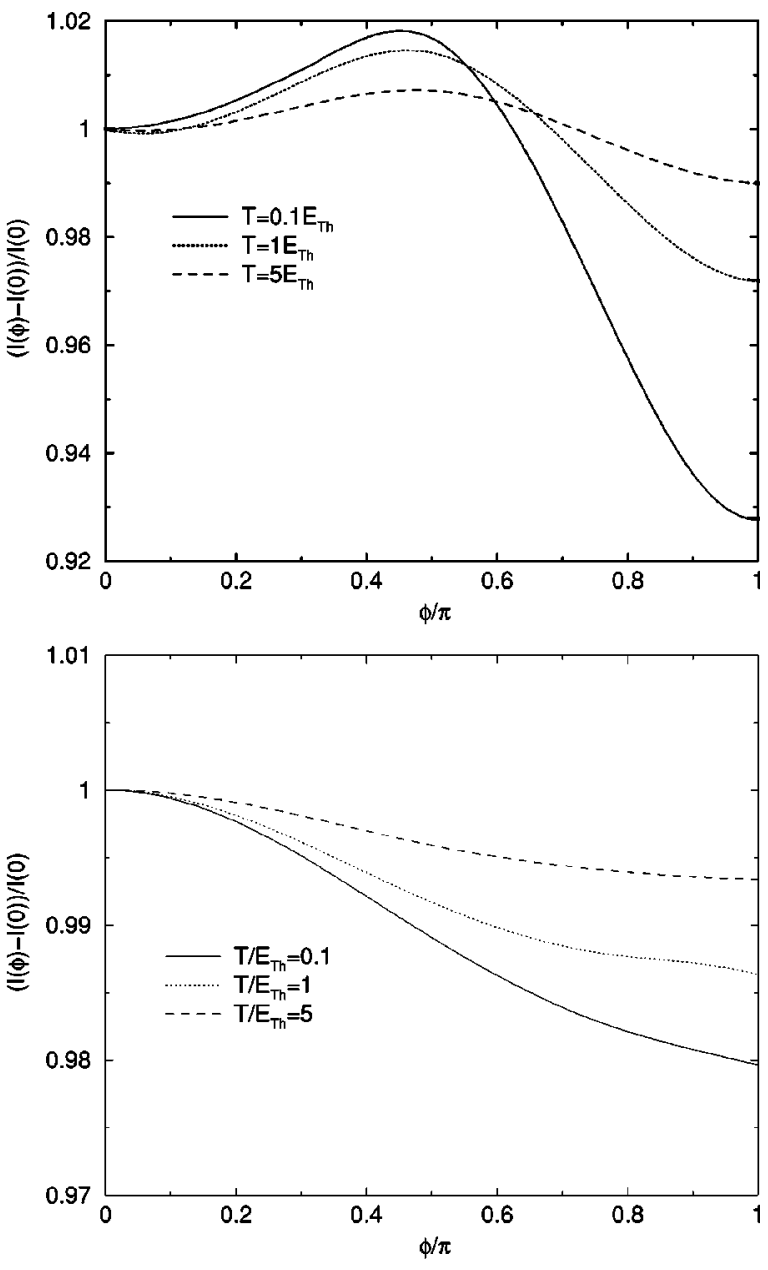

FIG. 5. Normalized current oscillations $[I(\phi)-I(0)] / I(0)$ at different temperatures for voltages $V / E_{\mathrm{Th}}=8.5,9.5,10.5,11.5$. 


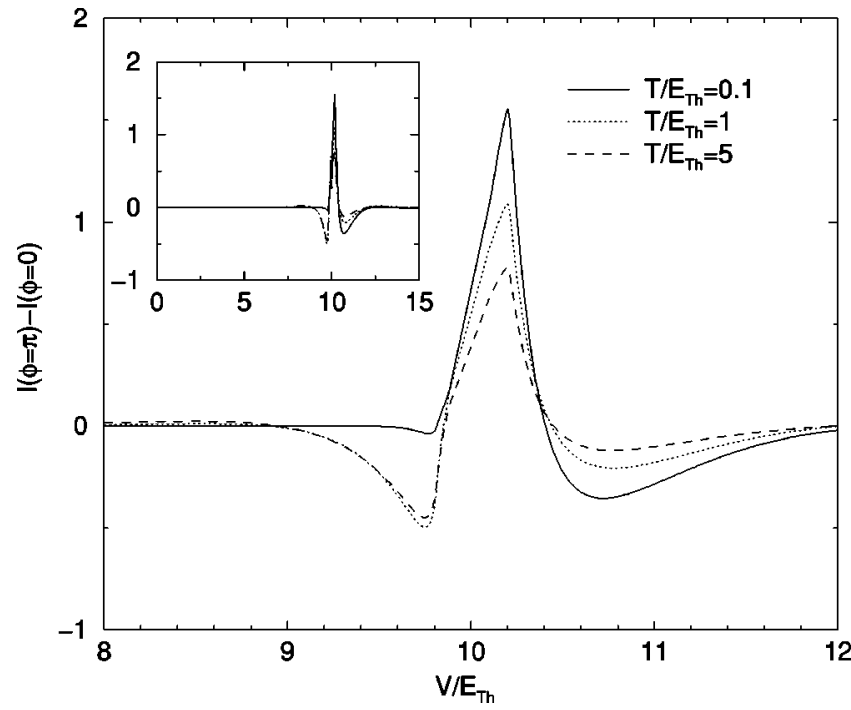

FIG. 6. Amplitude of the ( $\phi_{0}$-periodic) current oscillations as a function of the voltage. The inset shows the full structure.

$\approx V$, which will be achieved at intermediate $\phi$. Due to symmetry reasons, this does not only result into a phase shift, but into an intermediate maximum. Comparing $\phi=0$ and $\phi$ $=\pi$, one finds that depending on the particular voltage, there is a competition of the sharpness of the induced gap at $\phi$ $=0$ increasing on the current above the gap edge but decreasing it below the gap edge, which have to be traded off and, e.g., in Fig. 5, lower left, lead to a higher current at $\phi$ $=0$. At $V>\Delta+E_{g}^{\max }$, both peaks in the DOS contribute to the current, which is again leads to a zero-phase maximum, lower right.

A similar multitude of structures was observed in the $G(\phi)$ in the interferometer studied in the last section in the experiments by, e.g., by Antonov et al., see Ref. 9. In that paper, the conductance of an Andreev interferometer as probed through normal tunneling contacts was investigated. For technical reasons, small pieces of aluminum had to be deposited at the site of the barriers, which may become superconducting, rendering the structure a superconducting rather than a normal tunneling contact. As a result, there have been oscillations with intermediate maxima observed under certain bias conditions, which are compatible with our predictions. ${ }^{20}$ The oscillation amplitude, see Fig. 6, shows a remarkable peak structure. In the experiments, ${ }^{13}$ this effect will be washed out due to the two-dimensional geometry, however, a pronounced splitting of the conductance peak around $\Delta$ is observed. Remarkably and in agreement with Ref. 13, the oscillation amplitude in Fig. 6 only depends weakly on temperature, although we would have expected a strong $T$ dependence at least of the subgap peak. This observation in agreement with the experiments and makes it a likely explanation of the observed peak splitting. Our predictions can be studied in a more genuine setup like in the inset of Fig. 4, which is also remarkable to another reason: The attached tunneling contacts cool the distribution function in the normal metal by removing quasiparticles. ${ }^{21}$ This should also influence the supercurrent between the other two superconducting reservoirs in a way opposite to Ref. 22. Whether or not this also leads to $\pi$-junction behavior requires more detailed knowledge of the efficiency of the cooling. The experimental detection of the $\pi$ junction along the lines of Ref. 23 require detailed knowledge of the current-phase relations (Figs. 4 and 5) (in that terminology the control line), which is provided by our study.

Summary and conclusions. We have discussed the physics of proximity systems probed through a superconducting tunneling contact. We showed how these can be understood as junctions between two different superconductors separated by a tunneling barrier. This leads to a peculiar currentvoltage characteristic containing a step preceded by a small peak at $T>0$. We discussed the phase dependence of that current in a typical Andreev interferometer and outlined connections to existing and future experiments.

We would like to acknowledge useful discussions with A. D. Zaikin, G. Schön, T. M. Klapwijk, J. J. A. Baselmans, H. Weber, T. Heikkilä, O. Kuhn, and R. Taboryski. This work was supported by the DFG through SFB 195 and GK 284 and by the EU through the EU-TMR "Superconducting Nanocircuits.',
${ }^{1}$ P.G. de Gennes, Rev. Mod. Phys. 36, 225 (1964).

${ }^{2}$ Special issue of Superlattices Microstruct 25, 627 (1999), edited by P.F. Bagwell, and references therein.

${ }^{3}$ D. Estève, in Mesoscopic Electron Transport, edited by L.L. Sohn et al. (Kluwer, Dordrecht, 1997).

${ }^{4}$ C.J. Lambert et al., Phys. Rev. B 55, 6015 (1997).

${ }^{5}$ S. Guéron et al., Phys. Rev. Lett. 77, 3025 (1996).

${ }^{6}$ A.A. Golubov and M.Yu. Kupriyanov, J. Low Temp. Phys. 70, 83 (1988)

${ }^{7}$ W. Belzig et al., Phys. Rev. B 54, 9443 (1996).

${ }^{8}$ F. Zhou et al., J. Low Temp. Phys. 110, 841 (1998).

${ }^{9}$ V.N. Antonov, H. Takayanagi, F.K. Wilhelm, and A.D. Zaikin, Europhys. Lett. 50, 250 (2000).

${ }^{10}$ A.F. Volkov and A.V. Zaitsev, Phys. Rev. B 53, 9267 (1996).

${ }^{11}$ K.K. Likharev, Dynamics of Josephson Junctions and Circuits
(Gordon and Breach, New York, 1986).

${ }^{12}$ M. Tinkham, Introduction to Superconductivity, 2nd ed. (McGraw-Hill, Singapore, 1996).

${ }^{13}$ J. Kutchinsky et al., Phys. Rev. B 56, R2932 (1997).

${ }^{14}$ W. Belzig et al., Superlattices Microstruct. 25, 1251 (1999).

${ }^{15}$ A.F. Volkov et al., Superlattices Microstruct. 25, 647 (1999).

${ }^{16}$ A.A. Golubov et al., Phys. Rev. B 51, 1073 (1995).

${ }^{17}$ A. Barone and G. Paterno, Physics and Applications of the Josephson Effect (Wiley, New York, 1982).

${ }^{18}$ A.A. Golubov et al., Phys. Rev. B 55, 1123 (1997).

${ }^{19} \mathrm{H}$. Weber (private communication).

${ }^{20}$ V.N. Antonov (private communication).

${ }^{21}$ M.M. Leivo et al., Appl. Phys. Lett. 68, 1996 (1996)

${ }^{22}$ A. Morpurgo et al., Appl. Phys. Lett. 72, 966 (1998).

${ }^{23}$ J.J.A. Baselmans et al., Nature (London) 397, 45 (1999). 